\title{
Applying a CSA Scale to the Evaluation of Spatial Operation Ability for Use of a Control-Display Device
}

\author{
Chun-wei Chen ${ }^{1)}$, Chang-franw Lee ${ }^{1)}$ and Chien-cheng Chang ${ }^{3)}$ \\ 1) National Yunlin University of Science and Technology, Taiwan, R. O.C. \\ 2) Department of Industrial Design, Huafan University, Taiwan, R. O. C.
}

\begin{abstract}
The purpose of this study is to explore the feasibility of applying the CSA scale to measure the individual spatial operation ability of operators using a control-display device. Because competent operators may be able to reduce the possibility of serious accidents from the control room, it is important to be able to effectively measure the individual differences among operators. For this reason, we adopted a 2 (cognitive styles) $\times 2$ (control-display pairing patterns) splitplot design for our experiment. This paper examined the differences between the performances of participants with field-dependent (FD) cognitive styles and field-independent (FI) cognitive styles in control-display pairing tasks, and the correlation between CSA scores and performances. The results of the experiment indicated that the CSA scale is capable of distinguishing between employees in their capacity to operate control-display devices effectively. Therefore, it is suggested that together with other performance measurement criteria, the CSA scale should serve as a measurement criterion to help screen control room operators. This would reduce the occurrence of possible errors and help to improve control room safety. J Physiol Anthropol 27(6): 287-294, 2008 http://www. jstage.jst.go.jp/browse/jpa2

[DOI: 10.2114/jpa2.27.287]
\end{abstract}

Keywords: CSA scale, control-display device, spatial operation ability

\section{Introduction}

In this paper a control-display device refers to the type of control panel used in nuclear power plants, air traffic control towers, and the like. They are the communication channel between various machinery and operators in the control room and are often made up of a large number of control and display units (Sanders and McCormick, 1992). Often, the unsafe and inefficient operation of a complex control-display device is closely related to a disastrous event in the control room. It is therefore essential to explore how to maintain safe and efficient operation when using a control-display device. The topic of redesigning control-display devices has often been explored in the literature. However, for various reasons, such as the individual difference in abilities to operate a control-display device, not every operator may be able to safely and effectively operate one. Selecting the correct operators for a controldisplay device is an important issue for maintaining safe and efficient control room operation (Lhoir, 1984).

A control room operator refers to someone with a bachelor's degree in engineering or the physical sciences in a nuclear power plant, or a qualified air traffic controller in an air traffic control tower. Because the dexterity of the operators affects the possibility of disastrous events in the control room (Gray and Haas, 1984; Grosdeva and Montmollin, 1994; Seminara, 1980), any criteria that can be used to distinguish the difference between operators in the control room should be explored and listed for possible use in the evaluation of their ability to operate a control-display device. Establishing the appropriate criteria will help select suitable operators and reduce the possibility of a serious incident as a result of the improper operation of a control-display device.

In this study, Cognitive Styles Analysis (CSA) is suggested to help assess individual spatial operation ability in terms of operating a control-display device. Proposed by Riding and Cheema (1991), the CSA scale is an effective evaluative tool for measuring individual spatial cognitive ability in fielddependent-independent (FDI) cognitive styles, which is the human characteristic of separating a target from its contextual surroundings in a spatially misleading condition where the target is hidden in its contextual environment, making it hard to be identified (Witkin et al., 1962). When based upon a computerized administering system, the CSA is easier to use and is more valid (Redmond et al., 2007) than the traditional test of FDI cognitive styles. For this reason, the CSA has been widely used to measure a person's spatial operating abilities when processing spatial information.

However, we are short on auxiliary measurement criteria for the measurement of the individual spatial operation abilities needed to control a control-display device. Currently, the 
measurement criteria for the differences between operators when operating a control-display device are mainly composed of physical ability, mental ability, and personal characteristics (Bortolussi et al., 1986; Hayashi et al., 1990; Hendy et al., 1990; Itoh et al., 1989; Pickup et al., 2005). Physical ability includes functional criteria such as sight, hearing, ability to respond, and health conditions, including mental status. These criteria are often represented by tables of physical functional conditions such as sight and hearing observation data. Mental ability includes intelligence and aptitude tests such as the Wechsler Adult Intelligence Scale (WAIS) and the General Aptitude Test Battery (GATB). As far as personal characteristics are concerned, scales such as the California Psychological Inventory (CPI) and Strong Interest Inventory (SII) are frequently used for testing potential control-display device operators. These scales indicate that most current auxiliary measurements deal with physical abilities and mental abilities and seem to lack a measurement for spatial operation ability. Therefore, in this study we will use the CSA scale for measuring the individual differences in spatial operation ability among operators when controlling a control-display device.

In this study, it is hypothesized that the CSA scale can distinguish the differences in individual spatial operation ability among control-display device operators. Cognitive Styles Analysis (CSA) is an effective tool for the measurement of FD and FI cognitive styles. With this scale, individuals are divided mainly into those with a field-dependent (FD) cognitive style and those with a field-independent (FI) cognitive style (Riding and Cheema, 1991). FD individuals tend to be susceptible to deceptive environment cues, particularly in tasks that require the individual to identify familiar elements in an unfamiliar context (the misleading context). On the other hand, FI individuals tend to be able to disregard deceptive environment cues, particularly in tasks requiring identification of familiar elements in an unfamiliar context (Pascual-Leone, 1984, 1987, 1989). According to the research of different cognitive styles and their performance differences (Witkin et al., 1962), the difference between the performance of FD and FI individuals depends upon the degree of interference the misleading context has on the target in the spatial target-separating task. When the target is blurred and it is hard to tell from its contextual surroundings (the misleading context), FD individuals are more likely to be hindered by the context, resulting in a longer reaction time and consequentially a poor performance. On the other hand, the performance of FI individuals is less likely to be affected by the misleading context. This explains why there are performance differences between FD and FI cognitive styles as well as in the relationships between spatial target-separating tasks and spatially misleading situations.

To control a control-display device requires the characteristics of being good at spatial target separating and not being influenced by a spatially misleading condition. Operating a control-display device is a spatial control (stimulus)-display (response) pairing task (a spatial S-R pairing task) (Fitts and Seeger, 1953). Because of the multiple pairwise connections of control and display units in a controldisplay device, operators must conduct a search task for the target display unit within the control-display device and manipulate a large number of control units and display units. This search and hunt task is conducted within a misleading context where the operator needs to separate the target display unit from multiple display units within the control-display device. In other words, the control task (the control-display pairing task) in the control-display device is basically a spatial target-separating task under a spatially misleading condition. Thus, there may be different spatial operation abilities required by FD and FI individuals to manipulate the control-display device. For example, FI individuals may perform faster without being influenced by the control-display pairing task, compared to FD individuals whose performance may be poorer in the control-display pairing task. This shows the feasibility of using the CSA scale to distinguish the individual spatial operation ability differences between operators of a control-display device.

The above descriptions show the importance, necessity, and feasibility of using the CSA scale in exploring the individual spatial operation ability of operators in the control room. Based on the CSA scale, the operators who participated in the experiment of this study were asked to conduct spatial controldisplay pairing tasks so that we could explore the performance differences between different cognitive styles and the relationships between performance and cognitive style. The results obtained from this study may help the selection process for finding operators suitable for a control room.

\section{Spatial control-display pairing tasks}

Spatial control-display pairing refers to the pairing between the physical arrangement in space of the controls and their associated displays (Sanders and McCormick, 1992). These pairing tasks are frequently seen in spatial routines where the control units match the relevant displays within the controldisplay device. Fitts and Deininger (1964) further divided spatial control-display pairing tasks into correspondent and non-correspondent patterns. A condition is called "correspondent" when the related display units and control units are consistent, i.e., a horizontally arranged (vertically arranged) control unit responds to a horizontally arranged (vertically arranged) display (compatible condition) and the required reaction time is short. Conversely, when the corresponding display units and control units are inconsistent, i.e., a horizontally arranged (vertically arranged) control responds to a vertically arranged (horizontally arranged) display (incompatible condition), and the reaction time is longer. This condition is called "non-correspondent." These two pairing patterns differ in the corresponding relationships between the control and the display units, i.e., the correspondent pattern conducts a consistent corresponding relationship between control and display units while the noncorrespondent pattern conducts an inconsistent corresponding 
relationship. They represent most of the spatial operations required using a control-display device.

This study used a simple correspondent and noncorrespondent spatial control-display pairing task to represent a spatial control-display pairing task matching experiment. The simple spatial control-display pairing tasks in this study were designed based on simple tasks in a control room, a concept proposed by Umilta et al. (1990). Based on Umilta et al. (1990) a simple spatial control-display pairing task refers to a spatial corresponding task performed on a control-display device simplified from a common control-display device. In such a task, an operator performs a pairing task on a simple device that is composed of a few control and display units, i.e., an operator conducts a right (left) control-right (left) display/right (left) control-left (right) display corresponding task on a device that is composed of only two control units and two display units. These descriptions show that a simple spatial control-display pairing task maintains the same corresponding relationships between the control and display units in a common spatial control-display pairing task. This indicates that a simple spatial control-display pairing task has the same type of compatibility as a common spatial control-display pairing task. Therefore, this study used simple spatial controldisplay pairing tasks and a correspondent and noncorrespondent pairing task, to match the experiment.

\section{CSA scale and FDI cognitive styles}

Field-dependent-independent (FDI) cognitive styles refer to the two contrasting manners of processing spatial information, which are positioned along a continuum running from the extreme FD cognitive style to the extreme FI cognitive style (Witkin et al., 1962; Pascual-Leone, 1995). According to Pennings (1988) and Warber (1989), FD individuals use a holistic strategy to correspond to the spatial features of the schema (a form of mental imagery imagined from the referenced message) and the target message, so as to separate the target from its contextual surroundings. FI individuals, on the other hand, use an analytic strategy to correspond to the spatial features of the schema and the target message and to split the target from its contextual surroundings. Because it is easier for those individuals using the analytic strategy to spot the differences between referenced messages and the target signal, they are less likely to be influenced by the noises in the context (spatial features not related to the target signal). Therefore, FI individuals can separate the target quickly, and complete the spatial target-separating task in a short period of time. On the other hand, it is not easy for individuals using the holistic strategy to tell the difference between the referenced messages and the target signal. Hence, they are more likely to be influenced by the noise in the context and will take longer to finish the task of target separating. As a result, FD and FI cognitive styles are used as patterns of cognitive styles in this study.

Cognitive Styles Analysis (CSA) is an effective tool that was developed from the traditional test of FDI cognitive styles, such as the Embedded-Figure test proposed by Witkin et al. (1972, 1982). According to Pascual-Leone et al. (1994), these tests have a common underlying concept: a spatially misleading situation is used for all the questions in a test. Pascual-Leone et al. pointed out that the so-called spatially misleading situation indicates a task environment where the target signal is hidden in the spatial context, making it hard to be separated from the spatial context. The figure-separating task in Witkin's Embedded-Figure test and the figure-matching condition in the CSA scale are examples of spatially misleading situations. In these tests, the analytic strategy makes it easier for FI individuals to spot the target message, resulting in a shorter task time. On the other hand, FD individuals will spend a longer time completing the spatial target-separating task. In light of this, it is possible to tell the cognitive style of a specific individual based on the length of the task time in the test with a misleading context. The CSA has been widely used to measure FDI cognitive styles as a substitute for the traditional Embedded-Figure test due to its ability to be computerized, as well as to the validity of the test. For this reason, the CSA is used as a tool for measuring FDI cognitive styles. A CSA score above 1 indicates an FD cognitive style, while a score below 1 indicates an FI cognitive style (Riding and Cheema, 1991).

\section{Hypotheses}

The experiment and inferences in this study are conducted on the basis of the following two hypotheses: (1) FD and FI participants have different spatial operating abilities in controlling control-display devices, i.e., FI individuals have better performance than FD individuals in spatial controldisplay pairing tasks, and are not influenced by the complexity of the tasks (2) There is a specific relationship between CSA scores and the performance criteria of the spatial controldisplay pairing task, such as the corresponding task time / error times, i.e., there is a highly positive relationship between the CSA scores and the performances of FDI participants

\section{Method}

\section{Experimental design}

A 2 (cognitive styles) $\times 2$ (spatial control-display pairing patterns) design is adopted in this study. The two independent variables are the cognitive styles and the spatial control-display pairing patterns, where the cognitive styles cover the FD and FI cognitive styles levels and the spatial control-display pairing patterns consist of correspondent and non-correspondent spatial control-display pairing tasks.

In the experiment, participants are asked to conduct two spatial control-display pairing tasks. The order of the tasks is randomly assigned to each participant using random numbers. For example, a subject may process the correspondent task first and then the non-correspondent task while the other may do this in the reverse order. 


\section{Participants}

Sixty control room operators (30 participants per cognitive style) from five control rooms (the air traffic control towers of Chiayi Airport and Taichung Airport in Taiwan, the control center of the CPC (Chinese Petroleum Corporation), TRA (Taiwan Railways Administration), and the THRA (Taiwan High-Speed Railways Administration)) were recruited to participate in the experiment. Each participant's cognitive style was measured by CSA (FD participants: mean score $=1.21$, $S D=0.33$; FI participants: mean score $=0.62, S D=0.28)$. Their mean age was 36.4 years $(S D=4.8)$. A CSA score above 1 indicates an FD cognitive style, while a score below 1 indicates an FI cognitive style (Riding and Cheema, 1991). All participants were healthy and claimed to be right-handed. Their mean age was $41.2 \mathrm{yr}$. $(S D=4.4)$.

\section{Experimental task}

The control-display pairing experiment consisted of correspondent and non-correspondent spatial control-display pairing tasks. In the correspondent spatial control-display pairing task, the right response key corresponds to the right stimulus, and the left response key corresponds to the left stimulus. Conversely, the reverse condition is seen in the noncorrespondent spatial control-display pairing task, i.e., the right response key corresponds to the left stimulus, and the left response key corresponds to the right target stimulus. In the experimental tasks, the stimuli appear randomly.

In this study, the experimental tasks were programmed in Visual Basic. The screen background was black. The positions of the stimuli were outlined using two thin $3 \mathrm{~cm} \times 3 \mathrm{~cm}$ white squares (the distance between the two squares was $4 \mathrm{~cm}$ ). The stimuli appeared randomly, six times in two squares with a 2.5 $\mathrm{cm} \times 2.5 \mathrm{~cm}$ yellow square background.

While performing tasks in the experiment, each participant was asked to only use the index finger of their right hand, the dominant hand, to press the control button. In each trial, the push of the response key, whether correct or wrong, would automatically trigger the next trial with a pause of $0.3 \mathrm{sec}$.

\section{Equipment}

The experimental tasks in this study were simulated by Visual Basic and performed on a Notebook (Compaq Evo N610v). The participants used a two-key control unit (Lumina LP-400) to respond to the stimulus on the display. The distance between the display and the participant's eye was approximately $40 \mathrm{~cm}$.

\section{Experimental procedure}

The experiment was divided into two stages: participant sampling and spatial control-display pairing experiment. Before conducting the tasks in stage 2, participants were classified according to their CSA scores in stage 1.

\section{Stage 1: participant sampling}

Before the experiment, sixty male control room operators from five control rooms were recruited to participate in the experiment. If an operator was willing to participate in the experiment, he was given the CSA test. The CSA scale measured the cognitive style of each participant. Twelve participants were collected from each control room, amounting to a total of 60 participants for the experiment.

\section{Stage 2: the spatial control-display pairing experiment}

The participants from Stage 1 were then recruited to participate in the spatial control-display pairing experiment in Stage 2 .

1. They all received a briefing and a tutorial introducing the spatial control-display pairing experiment. The tutorial was repeated until each participant was familiar with the procedure.

2. To ensure that the participants understood the experimental procedure, each participant was asked to verbally repeat the way the test was to be conducted. After the tutorial, there was a 2-minute break.

3. The pushing of the enter button started the correspondent pairing tasks (6 trials) and the noncorrespondent pairing task ( 6 trials). In each trial, the stimulus appeared randomly on left or right locations on the screen. In the correspondent pairing task, a participant had to press the key that corresponded to the relevant stimulus on the screen, i.e., he had to press the left (right) key to respond to the stimulus that appeared randomly on the left (right) location on the screen. Conversely, a participant had to press an inconsistent key to respond to the relevant stimulus on the screen for a non-correspondent pairing task, i.e., he had to press the right (left) key to respond to the stimulus that appeared randomly on the left (right) location on the screen. In each trial, the push of the response key, whether it was correct or wrong, automatically triggered the next trial with a pause of $0.3 \mathrm{sec}$. If there were errors during the trial, it automatically jumped to the next trial. The number of errors and reaction times was recorded automatically.

4. During the test, only "Task starts" and "Task ends" were prompted on the screen. No messages were offered in order to avoid any interference with the test. A participant could not proceed to the next pairing task until he had completed the previous one.

\section{Performance criteria and statistical analysis}

The average reaction time and errors of the six tests in the control-display pairing task were used as the performance criteria in this study. The reaction time was defined as the time interval between the stimulus appearing on the screen and the response key being pressed.

In this study, significant variance analyses and regression analyses were performed to compare the performances of the FD and FI participants as well as the relationship between the CSA score of the FDI cognitive styles and the performances in the spatial control-display pairing tasks. A significance level of .05 was used to compare the performance means of the FD and 
Table 1 ANOVA analysis of FDI cognitive styles and control-display pairing patterns

\begin{tabular}{lcccc}
\multicolumn{1}{c}{ Variable } & $d f$ & $S S$ & $F$ value & $p$ \\
\hline FDI cognitive styles (A) & 1 & 623573.44 & 207.14 & $.00^{* *}:$ \\
Control-display pairing patterns (B) & 1 & 95687.11 & 31.76 & $.00^{* *}:$ \\
$\mathrm{A} \times \mathrm{B}$ & 1 & 10110.25 & 1.35 & .11 \\
\hline
\end{tabular}

$*: p<.05, * *: p<01$

Table 2 FD and FI participants' performances in the control-display pairing tasks

\begin{tabular}{|c|c|c|c|c|c|c|}
\hline \multirow{2}{*}{ Cognitive styles } & \multicolumn{2}{|c|}{ Correspondent } & \multicolumn{2}{|c|}{ Non-correspondent } & \multicolumn{2}{|c|}{$t$ test } \\
\hline & $\begin{array}{l}\text { Reaction time } \\
\text { (millisecond) }\end{array}$ & $\begin{array}{l}\text { Error } \\
\text { (times) }\end{array}$ & $\begin{array}{l}\text { Reaction time } \\
\text { (millisecond) }\end{array}$ & $\begin{array}{l}\text { Error } \\
\text { (times) }\end{array}$ & Reaction time & Error \\
\hline FI participants & $303.16(S D=57.28)$ & 1.2 & $319.40(S D=59.40)$ & 1.5 & $t=0.31$ & $t=0.52$ \\
\hline FD participants & $359.43(S D=44.47)$ & 1.4 & $394.75(S D=56.75)$ & 1.9 & $t=6.27^{*}$ & $t=3.21^{*}$ \\
\hline$t$ test & $t=3.14^{*}$ & $t=0.11$ & $t=15.10 * *:$ & $t=4.13^{*}$ & & \\
\hline
\end{tabular}

$*: p<.05, * *: p<.01$

FI participants. Moreover, the Pearson correlation coefficient (r) was used to examine the relationship between the CSA score of the FDI cognitive styles and the performances in the spatial control-display pairing tasks. Prior to significant variance analysis and regression analysis, all the experimental data were first normalized.

\section{Results}

Analysis of variance and correlation of cognitive styles and spatial control-display pairing patterns

The effects of two independent variables, cognitive styles and spatial control-display pairing patterns, were explored (Table 1). The analysis of variance indicated that both the FDI cognitive styles $(F(1,59)=207.14, p<.05)$ and the spatial control-display pairing patterns $(F(1,59)=31.76, p<.05)$ affected the performances of the participants. Furthermore, the Pearson correlation coefficient, $r=0.95$ (reaction time) $/ r=0.92$ (errors), demonstrated a highly positive correlation between the CSA score of the FDI cognitive styles and the performances in the spatial control-display pairing tasks.

The results of the ANOVA test indicated that the impact of the cognitive style on the operator's performance is larger than that of the spatial control-display pairing patterns. Moreover, the interaction between the cognitive style and the variables of the spatial control-display pairing pattern is low (interaction: $\mathrm{F}$ $(1,59)=1.35, p>.05))$. This finding indicates that the cognitive style is the dominant factor in the spatial control-display pairing tasks in the experiment.

\section{Effect of cognitive style on a participant's performance}

Table 2 shows the performance of FD and FI participants in the control-display pairing tasks. The experimental results of the performances of participants with two cognitive styles in the control-display pairing tasks (correspondent: FI (303.16 millisecond / 1.2 error times $)<$ FD (359.43 millisecond / 1.4 error times); non-correspondent: FI (319.40 millisecond / 1.5 error times) $<$ FD (394.75 millisecond / 1.9 error times)) indicates that the FI participants performed better in both the correspondent and the non-correspondent pairing tasks. In addition, it also shows that FD participants are influenced by the complexity of the tasks in accordance with the results that there is a significant difference between the performances of the FD participants in different patterns of control-display pairing tasks (reaction time: $\mathrm{t}(59)=6.27, p>.05$; error times: $\mathrm{t}$ $(59)=3.21, p>.05)$. However, no significant performance differences were found between correspondent and noncorrespondent control-display pairing tasks for FI participants (reaction time: $\mathrm{t}(59)=0.31, p>.05$; error times: $\mathrm{t}(59)=0.52$, $p>.05)$. This result affirms the first hypothesis that FD and FI participants have different spatial operating abilities in controlling control-display devices.

In addition, Table 3 indicates that there is a highly positive relationship between the CSA scores and the performances of FDI participants (reaction time: correspondent FI: $r=0.93$, FD: $r=0.89$ / non-correspondent FI: $r=0.87$, FD: $r=0.82$; error: correspondent FI: $r=0.89$, FD: $r=0.86$ / non-correspondent FI: $r=0.90$, FD: $r=0.81$ ). This supports the second hypothesis that there is a specific relationship between scores of CSA and the spatial operation ability of the operators.

Effects of spatial control-display pairing patterns on a participant's performance

When comparing the performances of the two cognitive styles in Table 2, all participants, regardless of their cognitive style, performed significantly faster in corresponding spatial control-display pairing tasks than in non-corresponding spatial control-display pairing tasks. This indicates that the easier it is to operate a control-display device, the better the participant's performance. 
Table 3 Regression analysis of CSA score and performance

\begin{tabular}{cllll}
\hline & & \multicolumn{2}{c}{ Correspondent } & Non-correspondent \\
\hline Reaction time & CSA: $r=.95^{*}$ & FI: $r=.93^{*}$ & FD: $r=.89^{*}$ & FI: $r=.87^{*}$ \\
error & CSA: $r=.92^{*}$ & FI: $r=.89^{*}$ & FD: $r=.86^{*}$ & FI: $r=.90^{*}$ \\
\hline
\end{tabular}

$*: p<.01$

$r$ : Pearson correlation coefficient

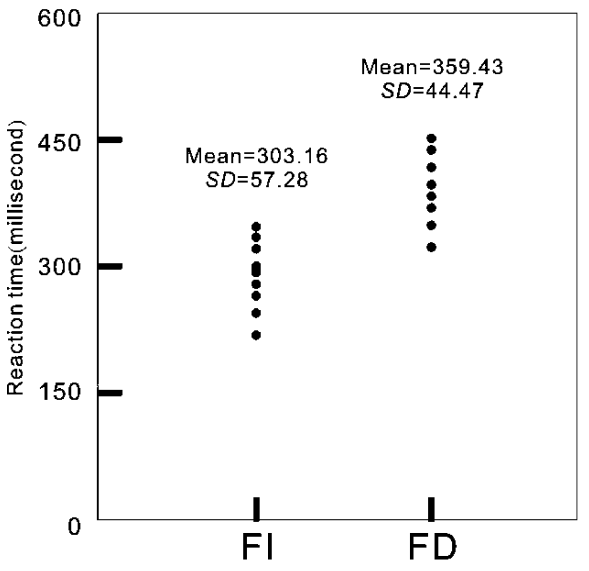

a. correspondent pattern

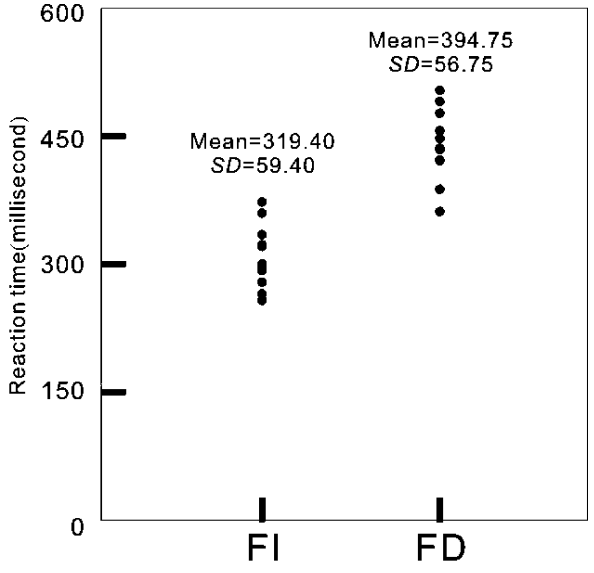

b. non-correspondent pattern

Fig. 1 The distribution of reaction time of FD and FI participants in both control-display pairing tasks.

\section{Discussion}

The feasibility of applying the CSA scale to measure the individual spatial operation ability of operators for use of a control-display device

The purpose of this study was to explore the feasibility of applying the CSA scale to measure the individual spatial operation ability of operators for use of a control-display device. The result of the experiment demonstrates that the CSA scale can be used to distinguish individual spatial operation abilities in controlling a control-display device.

First, the experimental result shows that FD and FI participants have different individual spatial operation ability of operating a control-display device. In Table 2, it can be seen that FI participants have better spatial operation abilities when performing both control-display pairing tasks. Table 2 shows that FI participants spent less time and had fewer errors than the FD participants when performing control-display pairing tasks. In addition, it shows that FI participants can efficiently and correctly perform the control-display pairing task, and are not influenced by the complexity of the tasks, implying that FD and FI operators have different spatial operation abilities. These results confirm that the CSA scale can distinguish individual spatial operation abilities in controlling a controldisplay device.

Moreover, the distribution analysis of the data of the reaction time in FD and FI participants (Fig. 1) also supports the affirmation that the CSA scale can distinguish individual spatial operation abilities in controlling a control-display device. Figure 1 shows that for both corresponding tasks, the overlap of the reaction time between FD and FI participants is low. It is evident that there is a gap between the performance of FD and FI participants in both the correspondent pairing task and the non-correspondent pairing task.

Finally, the highly positive correlation between the CSA scores and the operator's performance also supports the feasibility of measuring an individual's spatial operation ability of a control-display device using the CSA scale. In Table 3 it is shown that there is a specific relationship between CSA scores and spatial operation ability in the control-display pairing task. Table 3 shows that the higher the FI / FD scores the longer the reaction time / the larger the error times of the participants.

The extensional implications of applying the CSA scale in the screening of control room operators

The feasibility of applying the CSA scale in the screening of control room operators implies an extension of the measurement of the FDI cognitive styles. Currently, the measurement of FDI cognitive styles is used mainly in the evaluation and prediction of individual learning styles and learning achievement (Das et al., 2007; Gunther et al., 2007), and in the design and planning of the learning environment (Lee, 2007; Papanikolaou et al., 2006). The effectiveness of utilizing the CSA scale in the control room demonstrates that the measurement of FDI cognitive styles can be applied to other practical domains, particularly in those fields where 
spatial information processing is manipulated mainly during control-display pairing.

In addition, the individual difference in operating ability and adaptability to control-display devices found in this study helps to refine the conceptual framework in physiological anthropology. Sato (2005) stated that physiological anthropology is a steadily developing basic natural science. In the past, it has elucidated several theoretical concepts of human physiological characteristics. These conceptual frameworks have always been developed in a mosaic-like structure, and need to be refined. Sato investigated the development of the conceptual framework in physiological anthropology in order to strengthen the relationship among the different pieces of conceptual framework in physiological anthropology. By using the process of individual biology and through the process of population biology, Sato refined the conceptual framework of physiological anthropology in terms of technological adaptability, environmental adaptability, functional potentiality, and whole-body coordination. This refining must rely on the relationship between individual technological adaptability and operating a technological device. In this study, the feasibility of applying the CSA scale to measure the individual spatial operation ability of operators to use a control-display device demonstrates the individual technological adaptability to operate a technological device. As such, the result of the experiment in this study helps to refine the conceptual framework in physiological anthropology.

\section{Conclusion}

The results of this study demonstrate that the CSA scale is capable of distinguishing between employees in their capacity to operate control-display devices effectively. Therefore, it is suggested that the CSA scale is suitable as a measurement criterion in the screening of future control room operators, and should be included in the criteria for?their evaluation. This is likely to reduce the occurrence of possible errors and help to improve control room safety.

\section{References}

Bortolussi MR, Kantowitz, BH, Hart SG (1986) Measuring pilot workload in a motion base trainer: A comparison of four techniques. Appl Ergon 17: 278-283

Das JP, Janzen T, Georgiou GK (2007) Correlates of Canadian native children's reading performance: From cognitive styles to cognitive processes. J School Psychol 45: 589-602

Fitts PM, Seeger CM (1953) S-R compatibility: Spatial characteristics of stimulus and response codes. J Exper Psychol 46: 199-210

Fitts PM, Deininger RL (1964) S-R compatibility: Correspondence among paired elements within stimulus and response codes. J Exper Psychol: 48: 483-492

Gray LH, Haas PM (1984) Nuclear power plant control room operators' performance research. In Alluisi MJ, Groot SD,
Alluisi EA eds. Proceedings of the Human Factors Society 28th Annual Meeting. The Human Factors Society, Santa Monica, California, 239-241

Grosdeva T, Montmollin MD (1994) Reasoning and knowledge of nuclear power plant operators in case of accidents. Appl Ergon 25: 305-309

Gunther M, Evans G, Mefford L, Coe TR (2007) The relationship between leadership styles and empathy among student nurses. Nurs Outlook 55: 196-201

Hayashi Y, Tsukui I, Saito S (1990) The ergonomic evaluation of eye movement and mental workload in aircraft pilots. Ergonomic 33: 719-733

Hendy KC, Campbell EL, Laundry LN, Hamilton KM (1990) Review of workload: Measurement and prediction. Appl Ergon 21: 339-347

Itoh Y, Hayashi Y, Tsukui I, Saito S (1989) Heart rate variability and subjective mental workload in flight task validity of mental workload measurement using HRV method. In Smith MJ, Salvendy G eds. Work with Computers: Organizational, Management, Stress and Health Aspects. Elsevier, Amsterdam, 209-216

Lee J (2007) The effects of visual metaphor and cognitive style for mental modeling in a hypermedia-based environment. Interact Comput 19: 614-629

Lhoir J (1984) Selection and training of nuclear power plant operators. Office for Official Publications of the European Communities, Luxembourg, Report No EUR 7769 EN

Papanikolaou KA, Mabbott A, Bull S, Grigoriadou M (2006) Designing learner-controlled educational interactions based on learning/cognitive style and learner behavior. Interact Comput 18: 356-384

Pascual-Leone J (1984) Attention, dialectic, and mental effort: Toward an organismic theory of life stages. In Commons ML, Richards FA, Armon G eds. Beyond formal operations: Late adolescent and adult cognitive development. Praeger, New York, 182-215

Pascual-Leone J (1987) Organismic process model of neoPiagetian theories: A dialectical causal account of cognitive development. In Demetriou $\mathrm{A}$ ed. The neo-Piagetian theories of cognitive development: Towards integration. North-Holland, Amsterdam, 531-569

Pascual-Leone J (1989) Organismic process mode of Witkin's field-dependence/independence. In Globerson T, Zelniker T eds. Cognitive Style and Cognitive Development. Norwood, Ablex, NJ, 36-70

Pascual-Leone J (1995) Learning and development as dialectical factor in cognitive growth. Hum Dev 38: 338-348

Pascual-Leone J, Baillargeon RH (1994) Developmental measurement of mental attention. Int $\mathrm{J}$ Behav Dev 17: $161-200$

Pickup L, Wilson JR, Norris BJ, Mitchell L, Morrisroe G (2005) The Integrated Workload Scale (IWS): A new selfreport tool to assess railway signaler workload. Appl Ergon 36: 681-693 
Pennings A (1988) The development of strategies in embedded figures task. Int J Psychol 23: 65-78

Umilta C, Nicoletti R (1990) Stimulus-Response Compatibility. North-Holland, New York

Redmond JA, Parkinson A, Mullally, AP (2007) On the assessment of the test-retest of Riding's CSA: A commentary on Peterson, Deary, and Austin. Pers Individ Dif 43: 2306-2308

Riding RJ, Cheema, I (1991) Cognitive styles: an overview and integration. Educ Psychol 11: 219-215

Sanders MS, McCormick EJ (1992) Human Factors in Engineering and Design. McGraw-Hill, New York

Sato M (2005) The development of conceptual framework in physiological anthropology. J Physiol Anthropol Appl Hum Sci 24: 289-295

Seminara JL (1980) Human factors engineering enhancement of nuclear power-plant control rooms. Nuclear Safety 21: 351-363

Warber D (1989) The biological boundaries of cognitive styles: a neuropsychological analysis. In Globerson T, Zelniker T eds. Cognitive style and cognitive development. Norwood, Ablex Publishing Corporation, New Jersey, 11-35

Witkin HA, Dyk RB, Faterson HF, Goodenough DR, and Karp SA (1962) Psychological Differentiation. John Wiley and Sons, New York

Witkin HA, Goodenough DR (1982) Cognitive Styles: Essence and Origins-Field Dependence and Field Independence. International Universities Press, New York

Witkin HA, Oltman PK, Raskin E, Karp SA (1972) A Manual for the Embedded Figures Test, Children's embedded figures test, Consulting Psychologist. Palo Alto, CA

Received: March 24, 2008

Accepted: August 21, 2008

Correspondence to: Chunwei Chen, No.100, Gongming Rd., East District, Chiayi City 600, Taiwan, R.O.C.

Phone: +488-65-2783-550

e-mail: g9130807@yuntech.edu.tw 